Accepted version of: W. P. du Plessis, J. W. Odendaal, and J. Joubert, “Tolerance Analysis of Cross-Eye Jamming Systems," IEEE Transactions on Aerospace and Electronic Systems, vol. 47, no. 1, pp. 740-745, January 2010. Published version is available online at: http://ieeexplore.ieee.org/arnumber=5705705

(C) 2011 IEEE. Personal use of this material is permitted. Permission from IEEE must be obtained for all other uses, in any current or future media, including reprinting/republishing this material for advertising or promotional purposes, creating new collective works, for resale or redistribution to servers or lists, or reuse of any copyrighted component of this work in other works. 


\title{
Tolerance Analysis of Cross-Eye Jamming Systems
}

\author{
W. P. du Plessis, Member, IEEE, J. W. Odendaal Senior Member, IEEE, and J. Joubert Senior
}

Member, IEEE

\begin{abstract}
The matching required between the two directions through a retrodirective cross-eye jammer is considered using both the traditional phase-front analysis and an extended analysis. The design parameters to achieve a specified tracking error are derived and an optimal design is proposed. The results for the extended analysis show that the tolerances required to induce large angular errors in a monopulse radar are not as strict as the traditional analysis suggests.
\end{abstract}

\section{Index Terms}

Electronic warfare, electronic countermeasures, radar countermeasures, radar tracking, monopulse radar.

\section{INTRODUCTION}

Cross-eye jamming is an Electronic Attack (EA) technique that can be used to induce an angular error in the radar being jammed [1]-[9]. Angular deception is most often required in the final stages of an engagement where a platform is attempting to protect itself against radar-guided missiles. Cross-eye jamming has a number of benefits over other angular deception techniques, including the fact that it is effective against monopulse radars. A cross-eye jammer works by artificially recreating the worst-case glint error.

Glint is a phenomenon that arises when a radar is not able to resolve a number of scatterers and which can cause significant angular errors [10], [11]. However, a number of assumptions inherent in glint analyses are inaccurate for retrodirective cross-eye jamming [9]. An extended analysis of retrodirective cross-eye jamming that overcomes the limitations of glint analyses was recently published [8].

In this paper, closed-form solutions for the required amplitude and phase matching of the two directions through a retrodirective cross-eye jammer are derived. This is done for both the traditional phase-front analysis and the extended analysis. These models can be used to perform a system tolerance study to determine the matching required between the two paths through a retrodirective cross-eye jammer to achieve a specified angular error.

Closed-form solutions for the matching required from the two directions through a retrodirective cross-eye jammer are derived in Section II. Results are presented and compared in Section III, and a brief conclusion is provided in Section IV.

W. P. du Plessis is a Ph.D. student at the University of Pretoria, Pretoria, 0002, South Africa, and works for Defence, Peace, Safety and Security (DPSS), Council for Scientific and Industrial Research (CSIR), Pretoria, 0001, South Africa (e-mail: wduplessis@csir.co.za).

J. W. Odendaal and J. Joubert are with the Centre for Electromagnetism at the University of Pretoria, Pretoria, 0002, South Africa.

Manuscript received October 27, 2009; revised March 19, 2010. 


\section{Theoretical AnAlysis}

The effect of system tolerances on the performance of a retrodirective cross-eye jammer system is analysed below by determining the relative amplitude and phase shift of the two directions through a cross-eye jammer that achieve a specified angular error.

The basis of this analysis will be determining the angle where the monopulse indicated angle is zero. This angle will be referred to as the "settling angle." The settling angle is trivial to determine for the phase-front analysis considered in Section II-A, but as demonstrated in [8], does not exist for the extended analysis under certain conditions outlined in Section II-B.

The performance of a cross-eye jammer implementation can be characterised by an "angle factor" defined as

$$
G_{\theta}=\left|\frac{\theta_{s}}{\theta_{e}}\right|
$$

where $\theta_{s}$ is the settling angle and $\theta_{e}$ is half the angular separation of the jammer antennas from the radar's perspective as shown in Fig. 1. When the angle factor is greater than one, the apparent target created by a crosseye jammer will be outside the physical extent of the cross-eye jammer - an extremely desirable characteristic for a self-protection system - at all except very short ranges where the angular separation of the jammer antennas becomes large enough for the radar to resolve them.

The cross-eye gain is used extensively below and is given by [7]

$$
G_{C}=\frac{1-a^{2}}{1+a^{2}+2 a \cos (\phi)}
$$

where $G_{C}$ is the cross-eye gain, and $a$ and $\phi$ are the relative amplitude and phase shift of the two directions through the cross-eye jammer respectively. The value of the cross-eye gain is the same for $\phi=\pi+\phi_{0}$ and $\phi=\pi-\phi_{0}$, while only the sign of the cross-eye gain changes between $a=x$ and $a=1 / x$. Therefore, the discussion below is limited to positive values of cross-eye gain.

The angle factor and the magnitude of the cross-eye gain will be shown to be identical in the phase-front analysis case (the motivation for the definition of cross-eye gain), but not for the extended analysis. To avoid confusion, the term "angle factor" will be used when the relationship between settling angle and jammer antenna separation is intended, while the term "cross-eye gain" will only be used to refer to the relationship in (2).

\section{A. Phase-Front Analysis}

The indicated angle obtained using the phase-front analysis is given by [2]-[4], [6], [7]

$$
\theta_{i} \approx \theta_{r}+\theta_{e} G_{C}
$$

where $\theta_{i}$ is the monopulse indicated angle and $\theta_{r}$ is the angle from the radar's boresight to the centre of the cross-eye jammer as shown in Fig. 1.

The relationship between the cross-eye gain and the settling angle for phase-front analysis can be determined by substituting $\theta_{s}$ for $\theta_{r}$ and solving (3) for $\theta_{i}=0$ giving

$$
G_{C}=-\frac{\theta_{s}}{\theta_{e}}
$$

showing that the magnitude of the cross-eye gain is equal to the angle factor defined in (1) for the phase-front analysis. 


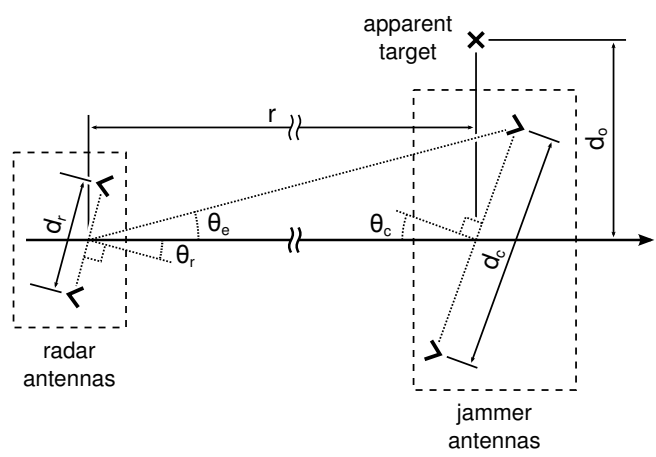

Fig. 1. The geometry of a cross-eye jamming scenario.

When $r \gg d_{c}, \theta_{e}$ can be written as [9]

$$
\theta_{e} \approx \frac{d_{c}}{2 r} \cos \left(\theta_{c}\right)
$$

where $d_{c}$ is the spacing of the jammer antennas, $r$ is the range to the jammer and $\theta_{c}$ is the jammer rotation as shown in Fig. 1. The settling angle can be written as

$$
\theta_{s} \approx \frac{d_{o}}{r}
$$

when $r \gg d_{o}$ where $d_{o}$ is the distance from the apparent target to the point in the middle of the jammer antennas as shown in Fig. 1. Substituting (5) and (6) into (4) gives

$$
\left|d_{o}\right| \approx G_{C} \frac{d_{c}}{2} \cos \left(\theta_{c}\right)
$$

which suggests that a cross-eye jammer induces a linear offset that does not vary with range [1], [7]. This property of cross-eye jamming is particularly valuable because a fixed angular offset would not cause a missile to miss its target [1].

Relationships between $a$ and $\phi$ that give a specified angle factor can now be derived by solving (2) for $a$ to give

$$
a=\frac{-G_{S} \cos (\phi) \pm \sqrt{1-\left[G_{S} \sin (\phi)\right]^{2}}}{1+G_{S}}
$$

and for $\phi$ to give

$$
\phi= \pm \arccos \left[\frac{1}{2 a}\left(\frac{\left|1-a^{2}\right|}{G_{S}}-1-a^{2}\right)\right]
$$

where $G_{S}$ is the magnitude of the specified cross-eye gain (which is identical to the angle factor for the phase-front analysis). Substituting $\phi=\pi \pm \phi_{0}$ into (9) gives

$$
\phi_{0}= \pm \arccos \left[-\frac{1}{2 a}\left(\frac{\left|1-a^{2}\right|}{G_{S}}-1-a^{2}\right)\right]
$$

which restates the phase result in terms of how close $\phi$ must be to $180^{\circ}$. These results can be used to determine the amplitude matching required to obtain a specified cross-eye gain for a given phase match, and the phase matching required to obtain a specified cross-eye gain for a given amplitude match.

Equations (8) to (10) give the matching required to achieve exactly the specified cross-eye gain $G_{S}$. The cross-eye gain will be larger than $G_{S}$ when $a$ is between the two solutions to (8) and when $\phi_{0}$ is between the 
two solutions to (10). This can be verified by evaluating (2) with $a=-G_{S} \cos (\phi) /\left(1+G_{S}\right)$ and $\phi_{0}=0$ $\left(\phi=180^{\circ}\right)$ to confirm that $\left|G_{C}\right|>G_{S}$ in these cases.

Equations (9) and (10) give the same result when $a=x$ and when $a=1 / x$. These two cases are equivalent because they both signify that gain of one direction through a cross-eye jammer differs from the gain in the other direction by the factor $x$. Equation (8) only gives values of $a$ with magnitudes less than 1, but the inverse of these results is clearly also valid. Values of $a$ with magnitudes greater than 1 can be obtained from (8) by using $-G_{S}$ instead of $G_{S}$.

A very important result that arises from (8) is that the range of values of $a$ for a given $\phi$ that gives a cross-eye gain magnitude of greater than $G_{S}$ does not include $a=1$ unless $\phi=180^{\circ}$. This supports the notion that designing for an amplitude mismatch between the two directions through a retrodirective cross-eye jammer allows a given angular error to be achieved with greater tolerance of phase mismatches between the two directions through the jammer [1], [4].

\section{B. Extended Analysis}

The monopulse indicated angle obtained using the extended analysis presented in [8]is given by

$$
\begin{gathered}
\tan \left[\beta \frac{d_{r}}{2} \sin \left(\theta_{i}\right)\right]=\frac{\sin (2 k)+\sin \left(2 k_{c}\right) G_{C}}{\cos (2 k)+\cos \left(2 k_{c}\right)} \\
k=\beta \frac{d_{r}}{2} \sin \left(\theta_{r}\right) \cos \left(\theta_{e}\right) \\
k_{c}=\beta \frac{d_{r}}{2} \cos \left(\theta_{r}\right) \sin \left(\theta_{e}\right)
\end{gathered}
$$

where $\beta$ is the free-space phase constant and $d_{r}$ is the spacing of the radar antennas as shown in Fig. 1. The forms of $k$ and $k_{c}$ in (12) and (13) differ from the forms provided in [8] because the assumption that $\theta_{e}$ is negligibly small was not made here.

Clearly the relationship between the settling angle and the jammer parameters is more complex for the extended analysis than for the phase-front analysis in the previous section. However, the problem of determining the settling angle is greatly simplified by noting that only the numerator of (11) has to be considered because the denominator cannot be infinite.

As noted in [8], there are conditions under which the monopulse indicated angle will never become zero, so the settling angle does not always exist. This occurs when the magnitude of the second term of the numerator in (11) is greater than 1 for all values of $\theta_{r}$ within the sum-channel's main beam. Determining whether this occurs requires a knowledge of how $k_{c}$ varies within the sum-channel's main beam.

The signal received in the sum channel is given by [8], [9]

$$
S_{r}=P_{r}\left(\theta_{r}-\theta_{e}\right) P_{c}\left(\theta_{c}-\theta_{e}\right) P_{r}\left(\theta_{r}+\theta_{e}\right) P_{c}\left(\theta_{c}+\theta_{e}\right) \times
$$

$$
\frac{1}{2}\left(1+a e^{j \phi}\right)\left[\cos (2 k)+\cos \left(2 k_{c}\right)\right]
$$

where $S_{r}$ is the received signal, and $P_{r}(\theta)$ and $P_{c}(\theta)$ are the patterns of the antenna elements comprising the radar and jammer respectively. From (11), it can be seen that the only portion of (14) that remains after monopulse processing is the trigonometric sum in the square brackets. The first nulls of the radar's sum-channel beam will thus be when the denominator of (11) is equal to zero. 
Substituting (12) and (13) into the denominator of (11) gives

$$
\cos (2 k)+\cos \left(2 k_{c}\right)=2 \cos \left[\beta \frac{d_{r}}{2} \sin \left(\theta_{r}+\theta_{e}\right)\right] \cos \left[\beta \frac{d_{r}}{2} \sin \left(\theta_{r}-\theta_{e}\right)\right]
$$

after some manipulation. The radar must not be able to resolve the two antennas comprising the cross-eye jammer, so $\theta_{e}$ must be much smaller than the value of $\theta_{r}$ where the sum-channel pattern becomes zero. This value can thus accurately be approximated by setting (15) equal to zero, assuming $\theta_{e}$ is negligible and solving for $\theta_{r}$ to give

$$
\theta_{r z} \approx \pm \arcsin \left(\frac{\lambda}{2 d_{r}}\right)
$$

where $\theta_{r z}$ is angle of the first null of the sum-channel pattern and $\lambda$ is the wavelength. This result is slightly conservative because a nonzero value of $\theta_{e}$ will make the first sum-channel pattern zeros slightly nearer boresight than suggested by (16).

Noting that $k_{c}$ reaches it maximum value when $\theta_{r}=0$ and substituting (16) into (13) gives

$$
\beta \frac{d_{r}}{2} \theta_{e} \sqrt{1-\left(\frac{\lambda}{2 d_{r}}\right)^{2}}<k_{c} \leq \beta \frac{d_{r}}{2} \theta_{e}
$$

in the sum-channel main beam. The square-root factor in (17) will be very close to 1 because $2 d_{r}$ is significantly larger than a wavelength in tracking radars, so it can be assumed that $k_{c}$ does not vary appreciably within the sum-channel main beam.

The monopulse indicated angle will never become zero in the sum-channel main beam when the magnitude of the cross-eye gain is greater than a specified minimum magnitude denoted $G_{S I}$ given by

$$
\begin{aligned}
G_{S I} & >\left|\frac{1}{\sin \left(2 k_{c}\right)}\right| \\
& \gtrsim \frac{1}{\sin \left(\beta d_{r} \theta_{e}\right)}
\end{aligned}
$$

where the simplest form of $k_{c}$ in (17) is used to give a simpler, but slightly optimistic result. Expanding $\theta_{e}$ using (5) allows (19) to be rewritten as

$$
G_{S I} \gtrsim \frac{\lambda}{\pi d_{r}} \times \frac{r}{d_{c} \cos \left(\theta_{c}\right)}
$$

assuming that the angular separation of the jammer antennas $\left(\theta_{e}\right)$ is much smaller than the radar beamwidth (determined by the factor $\beta d_{r}$ ). The values of $a, \phi$ and $\phi_{0}$ can now be obtained from (8) to (10) by using $G_{S I}$ for $G_{S}$.

The settling angle will exist when the above conditions are not satisfied. Again using the fact that $k_{c}$ is essentially constant in the sum-channel beam, expanding $k$ and $k_{c}$ under the assumption that $\theta_{e}$ is small, and substituting $\theta_{s}$ for $\theta_{r}$ gives

$$
\begin{aligned}
G_{C} & =-\frac{\sin (2 k)}{\sin \left(2 k_{c}\right)} \\
& \approx-\frac{\sin \left[\beta d_{r} \sin \left(\theta_{s}\right)\right]}{\sin \left(\beta d_{r} \theta_{e}\right)}
\end{aligned}
$$

which reduces to (4) when both $\theta_{s}$ and $\theta_{e}$ are much smaller than $\beta d_{r}$. This result is approximate and slightly conservative because the largest possible magnitude of the denominator was used. 


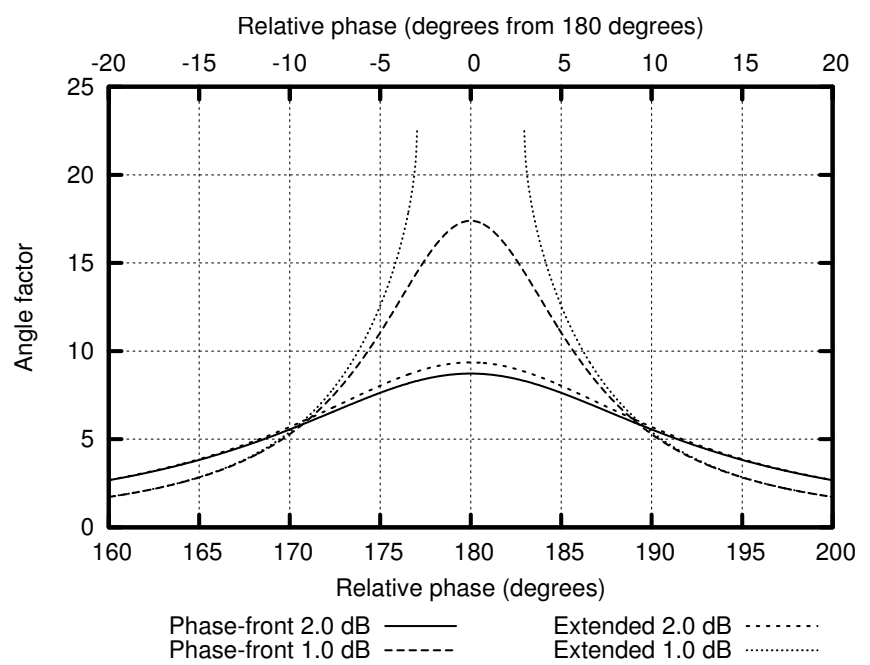

Fig. 2. The angle factor for a cross-eye jammer antenna element spacing of $5.0 \%$ of the radar beamwidth.

Noting that $G_{S}=\left|G_{C}\right|$ by definition and that $\left|\theta_{s}\right|=G_{\theta} \theta_{e}$ from (1), allows (22) to be rewritten as

$$
G_{S} \approx \frac{\sin \left[\beta d_{r} \sin \left(G_{\theta} \theta_{e}\right)\right]}{\sin \left(\beta d_{r} \theta_{e}\right)}
$$

allowing the values of $a, \phi$ and $\phi_{0}$ for a specified angle factor to be obtained from (8) to (10).

Unlike the phase-front case in (7), it is not possible to obtain a simple relationship between the linear error and either the cross-eye gain or the angle factor for the extended analysis. However, the results for the extended analysis converge to those of the phase-front analysis $\left(G_{S} \approx G_{\theta}\right)$ when the induced angular error $\left(G_{\theta} \theta_{e}\right)$ is much smaller than the radar antenna beamwidth (determined by $\beta d_{r}$ ).

\section{RESULTS AND COMPARISON}

The results derived above for the phase-front and extended analyses are compared in this section by considering a representative example.

The following parameters typical of a missile threat to a ship or aircraft [8] will be used:

- $10^{\circ}$ radar beamwidth $\left(d_{r}=2.54\right.$ wavelengths, and each radar element is a uniformly-excited aperture 2.54 wavelengths long),

- $1 \mathrm{~km}$ jammer range $(r=1 \mathrm{~km})$,

- $10 \mathrm{~m}$ jammer element separation $\left(d_{c}=10 \mathrm{~m}\right)$, and

- $30^{\circ}$ jammer rotation $\left(\theta_{c}=30^{\circ}\right)$.

The jammer antenna element angular spacing as seen by the radar is $0.50^{\circ}\left(\theta_{e}=0.25^{\circ}\right)$ which is $5.0 \%$ of the radar beamwidth. In all cases the relative amplitude and phase shift of the two directions through the jammer are provided on the figures.

The relationship between $a$ and $\phi$ is generally represented using a plot of the angle factor as a function of $\phi$ for a number of values of $a$ (e.g. [2], [6]) as shown in Fig. 2. Similar graphs are found in the glint literature (e.g. [11]) reflecting cross-eye jamming's origin. 


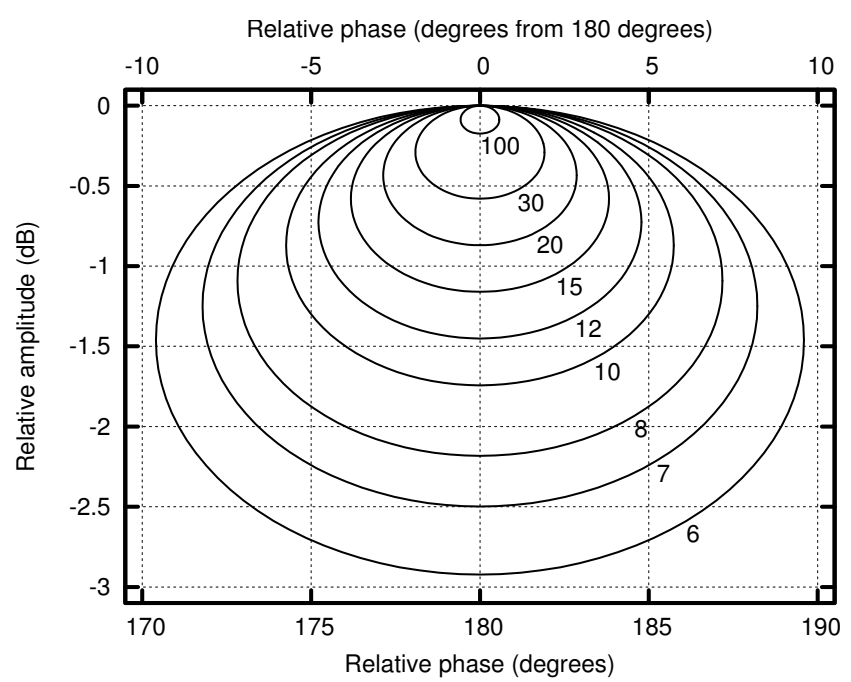

(a) Phase-front analysis.

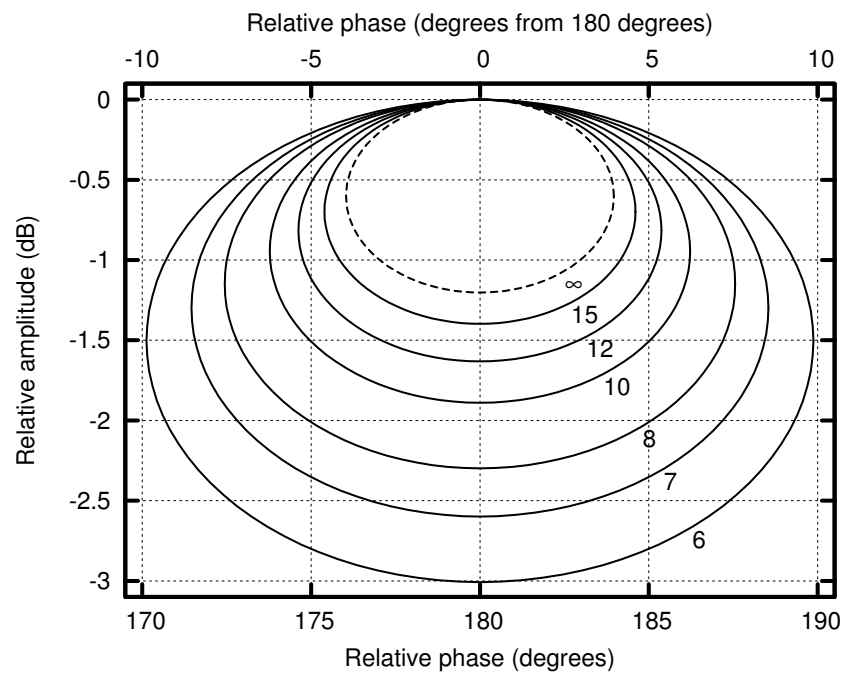

(b) Extended analysis.

Fig. 3. Contours of constant angle factor for a cross-eye jammer antenna element spacing of $5.0 \%$ of the radar beamwidth.

From Fig. 2, the agreement between the results using the two analyses is excellent when the relative phase shift of the two directions through the jammer is far from $180^{\circ}$. However, there is a significant difference between the phase-front and extended analyses when the relative phase shift approaches $180^{\circ}$ [8].

The plot of the results using the extended analysis with a relative amplitude of $1 \mathrm{~dB}$ ends abruptly at a relative phase shift of $2.95^{\circ}$ from $180^{\circ}$ because the settling angle does not exist in this case. This result agrees well with the value of $2.97^{\circ}$ obtained by determining $\phi_{0}$ from (10) using the cross-eye gain from (20).

While plots of the angle factor like Fig. 2 are valuable, contour plots based on (8) to (10) and (22) as shown in Fig. 3 are more useful. The specified angle factor will be achieved whenever the combination of $a$ and $\phi$ is on a constant angle-factor contour and exceeded whenever the combination of $a$ and $\phi$ is inside the contour. Fig. 2 is effectively a number of horizontal cuts through the contour plots in Fig. 3.

The contours in Fig. 3 are plotted using (8) so $|a| \leq 1$ making all the decibel values negative. As mentioned in Section II-A, $a=x$ and $a=1 / x$ represent equivalent cross-eye jammers, so the decibel values in Fig. 3 


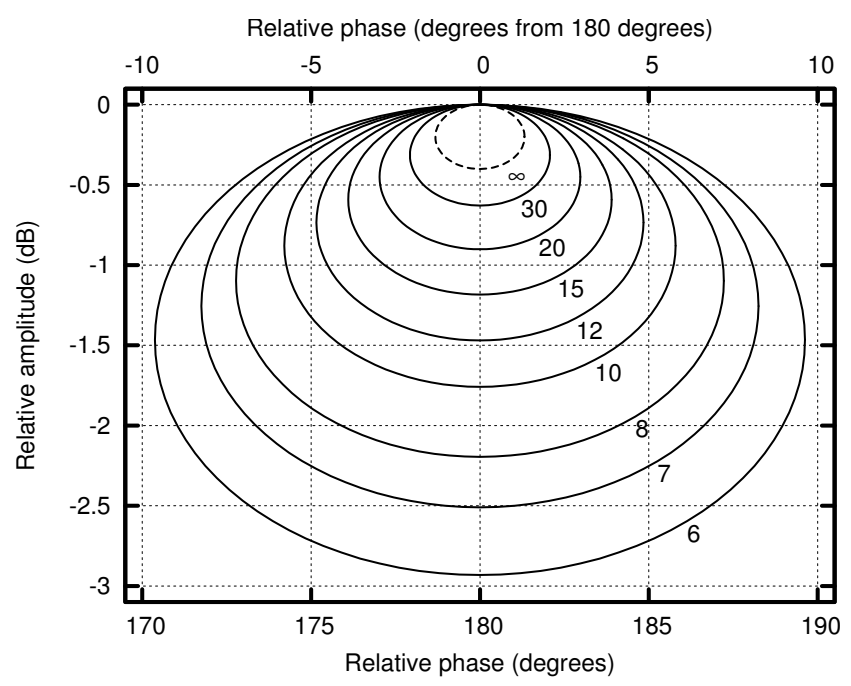

Fig. 4. Contours of constant angle factor according to the extended analysis for a jammer antenna element spacing of $1.65 \%$ of the radar beamwidth.

can be interpreted as being either positive or negative.

The fact that improving the amplitude match between the two directions through a cross-eye jammer can lead to a lower angle factor as discussed in Section II-A is clearly demonstrated in Fig. 3. This means that designing for a surprisingly large amplitude mismatch is actually the best way to achieve a specified angle factor [1], [4]. The results in Section II and the contour plots in Fig. 3 give clear quantitative and qualitative descriptions of this effect.

The amplitude or phase matching required to achieve a specified cross-eye gain for a given amplitude or phase match is considered in (8) to (10). However, optimum tolerances to both amplitude and phase variations for a specified cross-eye gain can be achieved by designing the system for the parameters at the centre of the relevant contour in Fig. 3. From (8) to (10), this requires a relative phase shift of $180^{\circ}$ and a relative amplitude of

$$
a=\frac{G_{S}}{G_{S}+1}
$$

where $G_{S}$ is determined either directly for the phase-front analysis, or from (20) or (23) for the extended analysis. As before, the inverse of the amplitude in (24) represents an equivalent solution.

The extended analysis shows that the tolerance requirements for the scenario considered are less strict than suggested by the phase-front analysis because contours in Fig. 3(b) are larger than those in Fig. 3(a). This allows greater parameter variations while still achieving the same minimum angular error in the radar being jammed.

The assumption that that the maximum angular error that can be caused by a cross-eye jammer is limited to $60 \%$ of the radar antenna's 3-dB beamwidth [1]-[5] (an angle factor of 6 for the scenario considered here) is also shown to be incorrect because curves with angle factors greater than 6 exist in Fig. 3(b).

As shown in (23), the extended analysis also depends on the angular separation of the jammer antennas $\left(\theta_{e}\right)$, so an additional set of contours at a range of $3 \mathrm{~km}$ is shown in Fig. 4. These curves show better agreement with the phase-front curves in Fig. 3(a) than the curves at shorter range in Fig. 3(b). Furthermore, the disagreement 
in both cases increases as the angle factor increases. This confirms Section II-B's assertion that the induced angular error (the product of the angle factor and the jammer antenna separation by definition in (1)) must be much smaller than the radar antenna beamwidth for the phase-front analysis to be accurate. This statement is more general than Vakin and Shustov's bound which requires $a \leq 0.9$ or $a \geq 1.1$ and $2 \theta_{e} \leq 10 \%$ of the radar's 3- $\mathrm{dB}$ antenna beamwidth for the phase-front analysis to be accurate [3], [4] because ignoring the relative phase means that Vakin and Shustov's bound is incomplete [9].

The traditional analyses of cross-eye jamming ignore the retrodirective implementation of cross-eye jamming and linearise a number of nonlinear effects [9]. These approximations are the cause of the differences between the widely-held assumptions about cross-eye jamming and the results presented here.

\section{Conclusions}

Closed-form solutions for the amplitude and phase matching between the two directions through a retrodirective cross-eye jammer required to achieve a specified angular error are presented. The results were derived for both the phase-front and extended analyses. Contour plots that give a clear graphical representation of the relationship between the matching and angular error are provided. Lastly, the combination of jammer parameters that achieves a specified angular error with the optimum tolerance to both amplitude and phase variations is provided.

The main conclusion of this work is that the tolerance requirements on a cross-eye jammer system are not as strict as the literature suggests. In particular, the maximum angular error is not limited to $60 \%$ of the radar antenna's 3-dB beamwidth as is widely believed.

\section{ACKNOWLEDGMENT}

The authors would like to thank the Armaments Corporation of South Africa (Armscor) and the National Research Foundation (NRF) for supporting this work.

\section{REFERENCES}

[1] P. E. Redmill, "The principles of artificial glint jamming (“cross eye")," Royal Aircraft Establishment (Farnborough), Tech. note RAD. 831, March 1963.

[2] L. B. Van Brunt, Applied ECM. EW Engineering, Inc., 1978, vol. 1.

[3] S. A. Vakin and L. N. Shustov, "Principles of jamming and electronic reconnaissance - volume I," U.S. Air Force, Tech. Rep. FTD-MT-24-115-69, AD692642, 1969.

[4] A. Golden, Radar Electronic Warfare. AIAA Inc., 1987.

[5] R. N. Lothes, M. B. Szymanski, and R. G. Wiley, Radar vulnerability to jamming. Artech House, 1990.

[6] A. I. Leonov and K. I. Fomichev, "Monopulse radar," U.S. Air Force, Tech. Rep. FTD-MT-24-982-71, AD742696, 1972.

[7] F. Neri, Introduction to electronic defense systems. Artech House, 1991.

[8] W. P. du Plessis, J. W. Odendaal, and J. Joubert, "Extended analysis of retrodirective cross-eye jamming," IEEE Transactions on Antennas and Propagation, vol. 57, no. 9, pp. 2803-2806, September 2009.

[9] W. P. du Plessis, "A comprehensive investigation of retrodirective cross-eye jamming," Ph.D. dissertation, University of Pretoria, 2010.

[10] J. W. Wright, "Radar glint - a survey," Electromagnetics, vol. 4, no. 2, pp. 205-227, January 1984.

[11] D. D. Howard, "Tracking radar," in Radar Handbook, 2nd ed., M. I. Skolnik, Ed. McGraw-Hill, 1990, ch. 18. 